\title{
DJ-1 can inhibit microtubule associated protein 1 $B$ formed aggregates
}

\author{
Zhiquan Wang ${ }^{1}$, Yu Zhang ${ }^{2}$, Shi Zhang ${ }^{2}$, Qianqian Guo ${ }^{3}$, Yuyan Tan² ${ }^{2}$ Xinyi Wang ${ }^{2}$, Ran Xiong ${ }^{2}$, Jianqing Ding ${ }^{*}$ \\ and Shengdi Chen ${ }^{1,2^{*}}$
}

\begin{abstract}
Background: Abnormal accumulation and aggregation of microtubule associated proteins (MAPs) plays an important role in the pathogenesis of neurodegenerative diseases. Loss-of-function mutation of DJ-1/Park7 can cause early onset of PD. DJ-1, a molecular chaperone, can inhibit $\alpha$-synuclein aggregation. Currently, little is known whether or not loss of function of DJ-1 contributes to abnormal MAPs aggregation in neurodegenerative disorders such as PD.

Results: We presented evidence that DJ-1 could bind to microtubule associated protein $1 \mathrm{~b}$ Light Chain (MAP1bLC). Overexpression of DJ-1 prevented MAP1b-LC aggregation in HEK293t and SH-SY5Y cells while DJ-1 knocking down (KD) enhanced MAP1b-LC aggregation in SH-SY5Y cells. The increase in insoluble MAP1b-LC was also observed in the DJ-1 null mice brain. Moreover, in the DJ-1 KD SH-SY5Y cells, overexpression of MAP1B-LC led to endoplasmic reticulum (ER) stress-induced apoptosis.
\end{abstract}

Conclusion: Our results suggest that DJ-1 acts as a molecular chaperone to inhibit MAP1B aggregation thus leading to neuronal apoptosis. Our study provides a novel insight into the mechanisms that underly the pathogenesis of Parkinson's disease (PD).

\section{Backgrounds}

PD is a common neurodegenerative disease which affects approximately $1 \%$ of individuals of 65 years and $5 \%$ of those 85 years or older. The featured pathological changes of PD are the selective and progressive loss of dopaminergic (DA) neurons as well as protein aggregation and Lewy body formation [1,2]. Lewy bodies mainly constitute of aggregated $\alpha$-synuclein protein and they also contain cytoskeletal components and other proteins. Although the role of protein aggregation in the pathogenesis of neurodegenerative diseases remains controversial, many studies have shown that protein aggregation contributes to neurodegeneration [2-5]. Failure to clear misfolded proteins leads to protein aggregation, which

\footnotetext{
* Correspondence: jqding18@yahoo.com; chen_sd@medmail.com.cn 'Laboratory of Neurodegenerative Diseases \& key Laboratory of Stem Cell Biology, Institute of Health Science, Shanghai Institutes for Biological Sciences, Chinese Academy of Science \& Shanghai Jiao Tong University School of Medicine. Shanghai 200025, China

${ }^{2}$ Department of Neurology \& Institute of Neurology, Ruijin Hospital Affiliated to Shanghai Jiao Tong University School of Medicine. Shanghai 200025, China

Full list of author information is available at the end of the article
}

may in turn lead to the pathogenesis of neurodegenerative diseases.

It has been reported that cytoskeletal proteins are involved in the pathology of neurodegenerative diseases [6,7]. For example, tau has been linked to both Alzheimer's disease (AD) and PD [8,9]. MAP1b has also been reported to participate in the pathogenesis of Fragile $\mathrm{X}$ syndrome [10] and Giant axonal neuropathy [11]. MAP1b plays a principal role in the development of the nervous system and is essential for normal development of the murine nervous system [12,13]. It has been reported that MAP1b co-localized with $\alpha$-synuclein in the Lewy body [14], which provides a hint that insoluble MAP1b may contribute to the pathogenesis of PD. Abnormal accumulation of MAP1B-LC leads to neuronal death in Giant Axonal Neuropathy (GAN) knockout (KO) mice [11]. So it is important to explore whether there is any link between MAP1b aggregation and PD pathogenesis.

Loss of function mutation of Park7/DJ-1 contributed to the pathogenesis of early-onset Parkinsonism [15]. Several PD-causing mutations have been identified

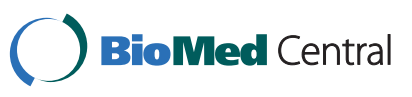


including exon deletions, truncations, homozygous and heterozygous point mutations, which are all predominantly in the loss of function manner [16]. DJ-1 belongs to the ThiJ/PfpI superfamily and expresses in both neurons and astrocytes $[17,18]$. DJ-1 could function as a molecular chaperone $[18,19]$ and inhibit the aggregation of $\alpha$-synuclein [20,21]. However, the exact role of DJ-1 in the cytotoxic process induced by MAPs aggregation is poorly understood. Here we reported that DJ-1 could directly bind to MAP1b-LC and inhibit its aggregation. Aggregation of MAP1b-LC was exacerbated when DJ-1 was deficient. Furthermore, we also showed that the excessive aggregation of MAP1b-LC could lead to apoptosis in DJ-1 KD SH-SY5Y cells. Therefore, DJ-1 may act as a molecular chaperone to suppress the neuronal death caused by protein aggregation.

\section{Results}

\section{DJ-1 interacted with MAP1b-LC}

MAP1b-LC has been shown to be a potential DJ-1 binding protein [22]. To study whether DJ-1 can interact with MAP1b-LC, GST-DJ-1 fusion protein and 6xHisMAP1b-LC were expressed in E. coli BL21 cells and purified respectively. The pull down assay showed that MAP1b-LC interacted with GST-DJ-1 but not with GST, suggesting that DJ-1 could bind to MAP1b directly in vitro (Figure 1A). Flag tagged MAP1b-LC and HA tagged DJ-1 were co-transfected into HEK293T cells for 36 hours. Cells were lysed and immunoprecipitated with either anti-Flag M2 beads or HA antibody-conjugated beads. The results showed that MAP1b-LC and DJ-1 could be immunoprecipitated reciprocally (Figure 1, B). Co-localization of DJ-1 and MAP1b-LC in HEK293t, SH-SY5Y cell lines (Figure 1, C and 1D) and cultured primary neurons (Figure 1F) was also observed. Furthermore, endogenous MAP1b-LC in the wild-type mice brain could also be immunoprecipitated by DJ-1 antibody-conjugated beads (Figure 1, E). These results all demonstrated that DJ-1 could form a complex with MAP1b-LC to regulate the physiological activities of MAP1b-LC.

\section{DJ-1 could inhibit the aggregation of MAP1b-LC}

It has been reported that MAP1b-LC is a component of cortical Lewy bodies [14], and abnormal accumulation of MAP1B-LC in the animal model of GAN could lead to neuronal death [11]. DJ-1 is thought to be a molecular chaperone that can inhibit the aggregation of $\alpha$-synuclein $[20,21]$. To explore whether DJ-1 can affect the aggregation of MAP1b, The Flag tagged MAP1b-LC construct was transfected into the HEK293t cells for 48 hrs. Cells were lysed and the lysates were separated into the detergent soluble and insoluble fractions [20]. These fractions were analyzed by SDS-PAGE/immunoblotting.
The results showed that MAP1b-LC formed aggregates were in the insoluble fraction (Figure 2A) and overexpressed DJ-1 decreased the insoluble MAP1b-LC (Figure 2A).

To further evaluate the effect of DJ-1 on MAP1b-LC aggregation, MAP1b-LC construct was transfected into HEK293t cells with either DJ-1 or the empty pcDNA3 vector as a control. We observed less MAP1b-LC aggregates in the DJ-1 overexpressed cells compared with the control (Figure 2B).

The relationship between DJ-1 and MAP1B-LC aggregation was also confirmed in the dopaminergic SH-SY5Y cells. Overexpression of DJ-1 decreased the insoluble MAP1b-LC fraction and inhibited the formation of MAP1b-LC aggregates when they were co-transfected into SH-SY5Y cells (Figure $2 \mathrm{C}, \mathrm{D}$ ). Moreover, overexpressed DJ-1 also decreased endogenous insoluble MAP1b-LC in SH-SY5Y cells (Figure $2 \mathrm{E}$ ). Taken together, these data revealed that DJ-1 could act as a chaperone to inhibit the abnormal aggregation of MAP1b-LC in both HEK293t cells and SH-SY5Y cells.

\section{Malfunction of DJ-1 exacerbated aggregation of MAP1b-LC}

L166P, the most common form of DJ-1 mutation, can prevent the dimer formation. The DJ-1 mutant is unstable and is degraded rapidly [23]. These observations have suggested that the DJ-1 mutation could be a loss-of-function mutation. To examine whether or not the DJ-1 mutation impairs the ability of DJ-1 to inhibit MAP1B-LC aggregation, Flag-MAP1b-LC was co-transfected into HEK293t or SH-SY5Y cells with either pEGFP-DJ-1 L166P or pEGFP vector. The results showed that L166P mutation of DJ-1 failed to suppress the accumulation of insoluble MAP1b-LC (Figure $3 \mathrm{~A}, \mathrm{~B}$ ). DJ-1 KD SH-SY5Y cell line was also established to further investigate whether the malfunction of DJ-1 could induce the aggregation of MAP1b-LC. Western blot results confirmed that DJ-1 was effectively knocked down in the DJ-1 shRNA stable cells compared with scramble shRNA control (Figure 4A). To examine the effect of down regulation of DJ-1 on the aggregation of MAP1b-LC, the Flag-MAP1b-LC was transfected into DJ-1 KD cells or scrambled control cells. The result showed that there was more insoluble Flag-MAP1b-LC in the DJ-1 KD cells compared with the controls (Figure 4B). Similarly, increased Flag-MAP1b-LC aggregation was also observed in the DJ-1 KD cell lines (Figure 4C). Furthermore, we also observed the increased endogenous insoluble MAP1b-LC (Figure 4D) and the endogenous MAP1b-LC aggregates was observed in DJ-1 KD SHSY5Y cells (Figure 4E).

The relationship between MAP1b-LC aggregation and DJ-1 deficiency was also studied in vivo. Sixmonth-old DJ-1 KO mice or wild type littermates were 


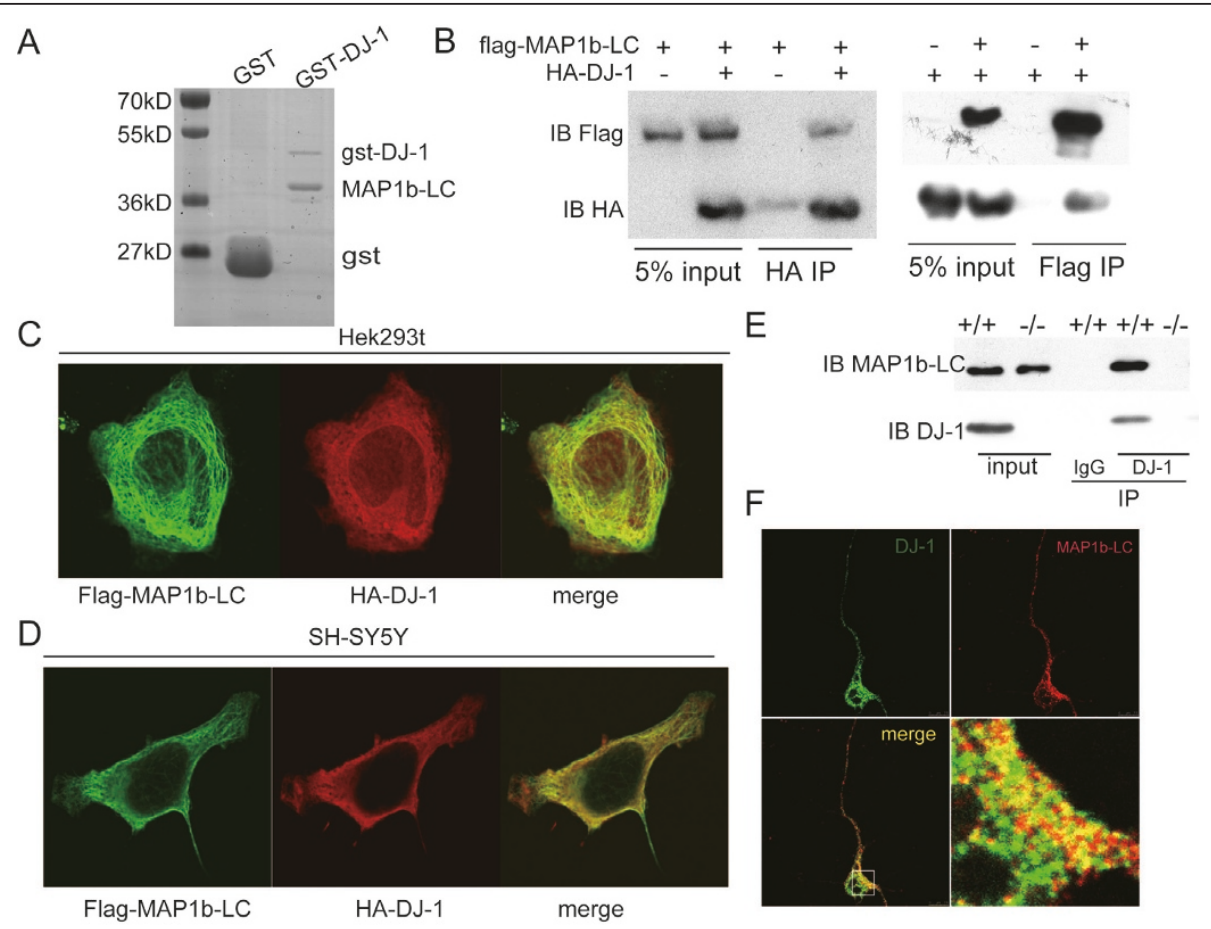

Figure 1 DJ-1 interacted with MAP1b light chain. A, GST-DJ-1 or GST was incubated with 6xhis-MAP1b-LC for 3 hrs and then was pulled down by GST beads, the beads were washed and SDS-PAGE followed by Coomassie blue staining was used to analyze the result. The result showed DJ-1 could bind to MAP1b-LC directly in vitro. B, Flag-MAP1b-LC and HA-DJ-1 were transfected into HEK293t cells for 36 hrs and the cell lysates were immunoprecipitated with Flag M2 beads or HA antibody conjugated beads. Both DJ-1 and MAP1b-LC were detected by western blot with HA or Flag antibody respectively. C and D, Co-localization of MAP1b-LC and DJ-1. Flag-MAP1b-LC and HA-DJ-1 were cotransfected into KEK293t (C) or SH-SY5Y cells (D) for 36 hrs before fixed and stained with rabbit anti-Flag and mouse anti-HA antibodies. E. The wild type or DJ-1 $\mathrm{KO}$ mouse brain lysates were immunoprecipitated with DJ-1 antibody, MAP1b-LC could be detected in the wild type mouse brain but not in that of the KO mouse. F, The primary cortical neuron was fixed and immuno-stained with DJ-1 and MAP1b-LC antibody.

used. The brain lysates of $3 \mathrm{KO}$ or wild type mice were extracted and separated into Triton-X100 soluble and insoluble components. The Western blot results showed an increase in insoluble MAP1b-LC in the DJ-1 KO mouse compared with that of the wild type (Figure 4F, G). Taken together, our results showed that DJ-1 abolishment enhanced MAP1b-LC aggregation both in vitro and in vivo.

\section{DJ-1 abolishment did not alter the ubiquitination of MAP1b-LC and the activity of proteasome}

MAP1b-LC is degraded through the ubiquitin proteasome system (UPS) and impairments of the UPS in the GAN-null mice may lead to the accumulation of MAP1b-LC [11]. DJ-1 has also been shown to form a complex with Pink1 and Parkin to promote degradation of unfolded or misfolded proteins [24]. Since failure of UPS has been thought to play a critical role in the pathogenesis of $\mathrm{PD}$, we explored whether ubiquitination of MAP1b-LC was altered in the DJ-1 KD cells. Flag-tagged MAP1b-LC was transfected into the DJ-1 KD SH-SY5Y cells or scrambled control cells. Cells were lysed and the lysates were immunoprecipated with anti-Flag antibody and probed with anti-ubiquitin antibody. The result showed that the ubiquitination of MAP1b-LC was unchanged when DJ-1 was knocked down (Figure 5A and 5B).

Down-regulation of DJ-1 can enhance the death of proteasome inhibitor-treated Neuro2A cells [25], so we attempted to assess whether DJ-1 deficiency may affect the proteasome activity. Proteasome chymotrypsin-like, caspase-like and trypsin-like activities were measured with succinyl-Leu-Leu-Val-Tyr-AFC, Z-LeuLeu-Glu-AMC and Boc-Leu-Arg-Arg-AMC, respectively [26]. The results did not show any significant difference between the proteasome activity of DJ-1 KD cells and that of scrambled control (Figure 5C). Using the same method, we did not find the impairment of proteasome activity in DJ-1 KO mice brain either (Figure 5D). These results suggest that the DJ-1 deficiency did not affect the activity of UPS and ubiquitination of MAP1b-LC. DJ-1 may work as a molecular chaperone to regulate the folding of MAP1b-LC but not its ubiquitination. 


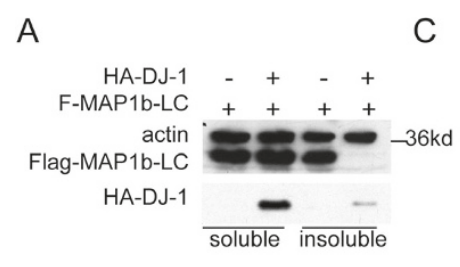

B

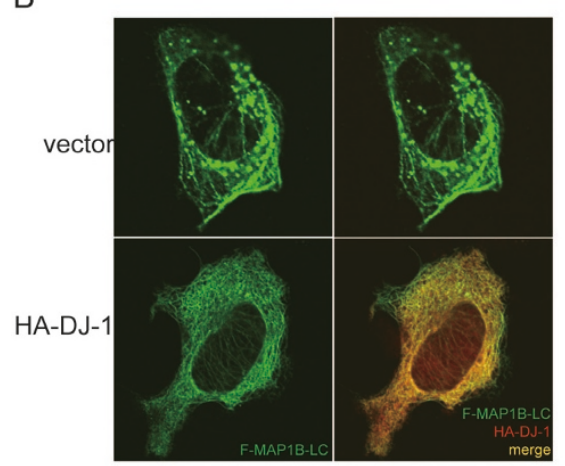

$\mathrm{E}$

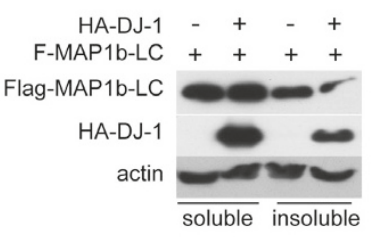

D

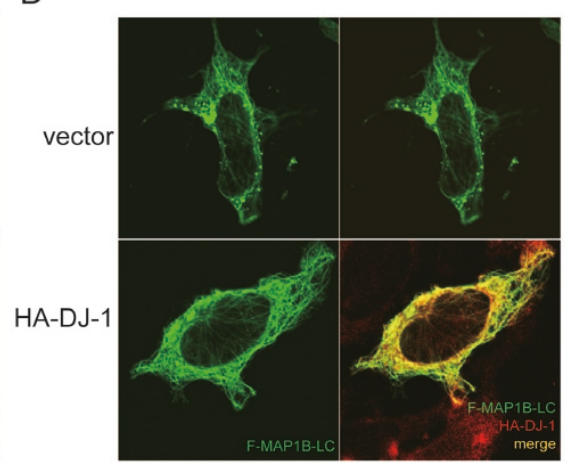

Figure 2 DJ-1 inhibited MAP1b-LC aggregation in HEK293t and SH-SY5Y cells. A, Flag-MAP1b-LC was co-transfected with HA-DJ-1 or pcDNA3 vector into HEK293t cells for 48 hrs before the cells were lysed and separated into Triton $x-100$ soluble and insoluble fractions. Western blot results showed overexpression of DJ-1 almost abolished the insoluble Flag-MAP1b-LC. B, Flag-MAP1b-LC and HA-DJ-1 or PCDNA3 were cotransfected into HEK293t cells for 48 hrs before the cells were fixed and immuno-stained with rabbit anti-Flag or mouse anti-HA antibodies. The most representative images were shown and the results showed the inhibition of MAP1b-LC aggregates in the HA-DJ-1 transfected cells. C, FlagMAP1b-LC was co-transfected with PCDNA3-HA-DJ-1or pCDNA3 into the SH-SY5Y cells and cultured for 48 hrs before the cells were lysed and separated into Triton X-100 soluble and insoluble fractions. Western blot results showed that overexpression of DJ-1 suppressed the insoluble Flag-MAP1b-LC. D, SH-SY5Y cells were transfected with Flag-MAP1b-LC and PCDNA3-HA-DJ-1 or pcDNA3 respectively. Forty-eight hours later the cells were fixed and stained with rabbit anti-Flag or mouse anti-HA. The results showed the inhibition of MAP1b-LC formed aggregates in the HA-DJ-1 transfected cells. E, pcDNA3-HA-DJ-1 or pcDNA3 was transfected into the SH-SY5Y cells for 48 hrs before the cells were lysed and separated into Triton $x-100$ soluble and insoluble fractions. Western blot results suggested overexpression of DJ-1 could suppress the endogenous insoluble MAP1b-LC.

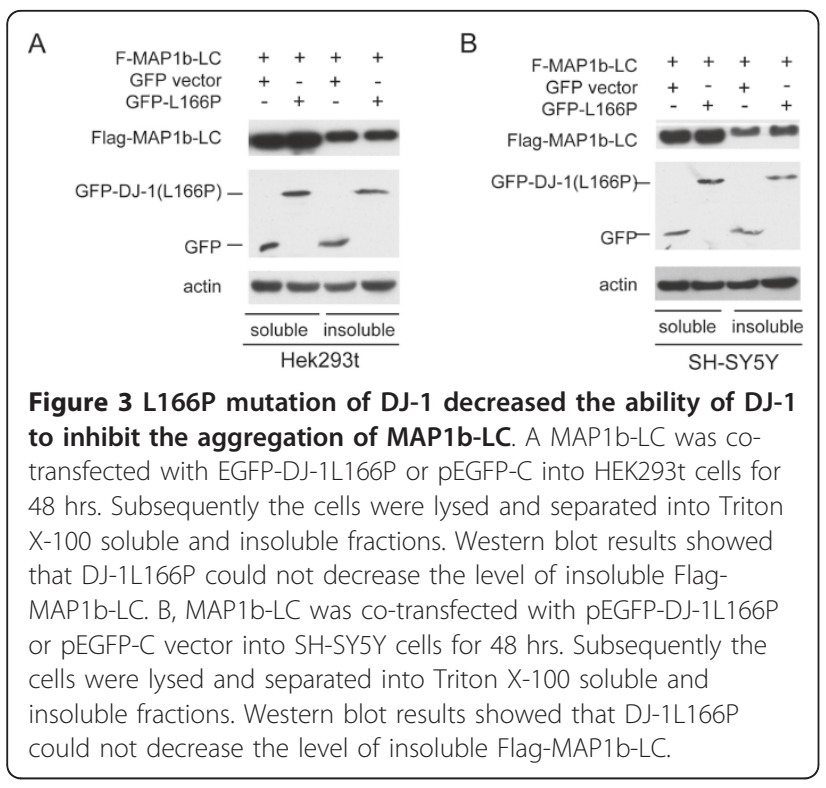

MAP1b-LC aggregation caused ER stress dependent apoptosis in the DJ-1 KD SH-SY5Y cells

Since protein aggregation is a major cause of neurodegeneration and malfunction of DJ-1 can lead cells to produce more aggregated MAP1b-LC, we next studied whether MAP1b-LC aggregation induced apoptosis of DJ-1 KD SH-SY5Y cells. We transfected the MAP1b-LC into DJ-1 KD SH-SY5Y cells or scrambled control, pDsRed2 being co-transfected with MAP1b-LC for the selection of the transfected cells. After 60 hours, the cells were harvested for the Annexin- $\mathrm{V}$ apoptosis assay. We observed more Annexin-V positive cells in the MAP1b-LC transfected group (Figure 6A, d) compared to that of the scramble cells (Figure 6A, c) (Figure $6 \mathrm{~B}$ ). Since apoptosis caused by protein aggregation is mainly through the ER stress dependent pathway [27], we examined the phosphorylated eIF2 $\alpha$, an ER stress marker. Phosphorylation of eIF $2 \alpha$ will only happen at the early stage of ER stress to counteract the insult and is 


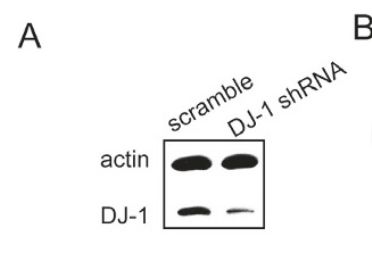

C

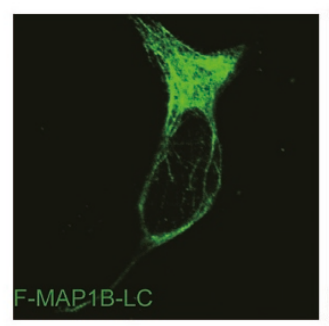

scramble shRNA

E

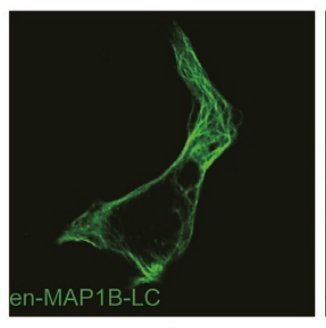

scramble shRNA
B

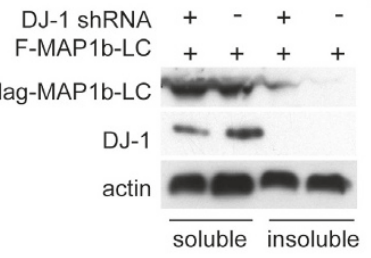

D

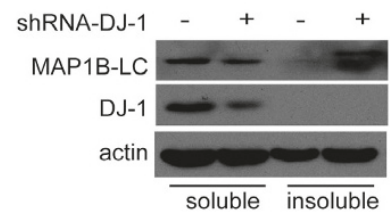

$\mathrm{F}$

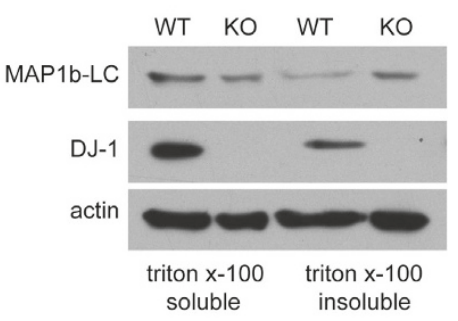

G

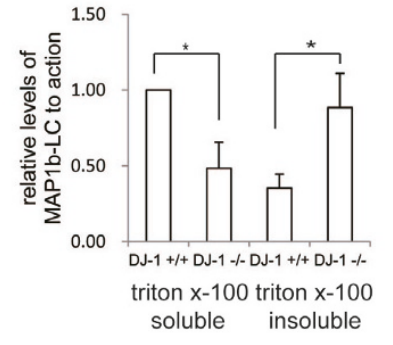

Figure 4 Loss of function of DJ-1 increased the aggregation of MAP1b-LC. A, DJ-1 shRNA vector or scramble shRNA vector was transfected into SH-SY5Y cells and selected with $300 \mu \mathrm{g}$ hygromycin. The stable clone was picked and amplified. Western blot result showed efficient downregulation of DJ-1. B, Flag-MAP1b-LC was transfected into DJ-1 shRNA or scramble SH-SY5Y cells for 48 hrs. Cells were lysed and separated into Triton X-100 soluble or insoluble components. The result showed increased insoluble MAP1b-LC in the DJ-1 KD cells. C, Flag-MAP1b-LC was transfected into DJ-1 shRNA SH-SY5Y or scramble shRNA cells for 48 hrs. Cells were immuno-stained with rabbit anti-Flag antibody. The results showed the enhancement of MAP1b-LC formed aggregates in DJ-1 KD cells. D, The total lysates of DJ-1 KD SH-SY5Y cells or scramble controls were separated into Triton X-100 soluble or insoluble fractions. Increased insoluble MAP1b-LC in DJ-1 KD cells was observed by Western blot. E, Endogenous MAP1b-LC was examined by immunofluorescence assay in DJ-1 KD SH-SY5Y cells or scramble controls. The MAP1b-LC formed aggregates increased in DJ-1 KD cells. F and G, DJ-1 deficiency intensified the formation of insoluble MAP1b-LC in vivo. F, The wild type or DJ-1 KO mice brain lysates were separated into Triton X-100 soluble or insoluble fractions. Western blot was used to analysis of MAP1b-LC distribution in the soluble or insoluble fractions. G, Quantification of relative levels of MAP1 b-LC (*, $p=0.03$ ).

thought to be protective for the cells from ER stress. However, when the insult continues, activated eIF $2 \alpha$ will be dephosphorylated and the protection will be abolished, leaving the cells to undergo apoptosis $[28,29]$. Our results showed that phosphorylated eIF2 $\alpha$ was much higher in MAP1b-transfected DJ-1 KD cells at 48 hrs after transfection compared with that in the scrambled control DJ-1 KD cells (Figure 7A). However, eIF2 $\alpha$ dephosphorylation in the MAP1b-LC transfected DJ-1 KD cells was increased at $60 \mathrm{hrs}$ after transfection (Figure 7B). These results showed that phosphorylation of eIF2 $\alpha$ was induced upon MAP1b-LC aggregation to protect the cells against ER stress and increased MAP1b-LC aggregation induced severer ER stress in DJ$1 \mathrm{KD}$ cells. However, the protection was abolished as the DJ-1 deficient cells failed to decrease the aggregated proteins in the DJ-1 KD cells, which finally led to ER stress induced apoptosis. Particularly, Salubrinal, the specific inhibitor of dephosphorylation of eIF2 $\alpha$ [28] suppressed the MAP1b-LC induced apoptosis in DJ-1 KD SH-SY5Y cells (Figure 7C and 7D). Taken together, these results suggested that excessive MAP1b-LC aggregation caused by DJ-1 ablation may induce apoptosis in the ER stress dependent manner.

\section{Discussion}

Mutations of $D J-1$ have been linked to early onset Parkinsonism. However, the molecular mechanism underlying the pathogenesis is still obscure. DJ-1 is thought to be a molecular chaperone and oxidative sensor, participating in both familial and sporadic PD $[20,30]$. Most researches have been focused on the anti-oxidative 


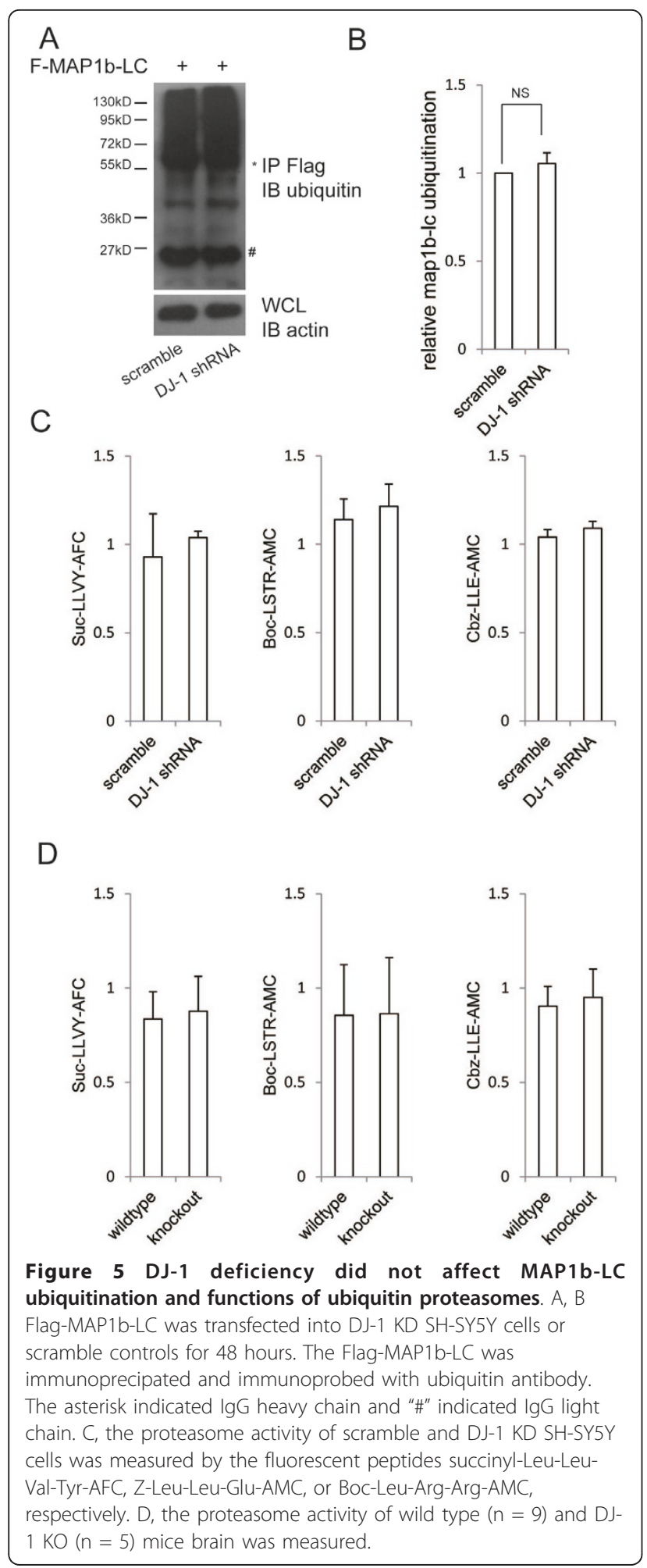

stress function of DJ-1 [31-35] but the molecular chaperone function of DJ-1 was hardly noticed [20]. Our results showed that DJ-1 interacted with MAP1b-LC both in vitro and in vivo. Furthermore, aggregates formed by overexpressed MAP1b-LC in HEK293t and

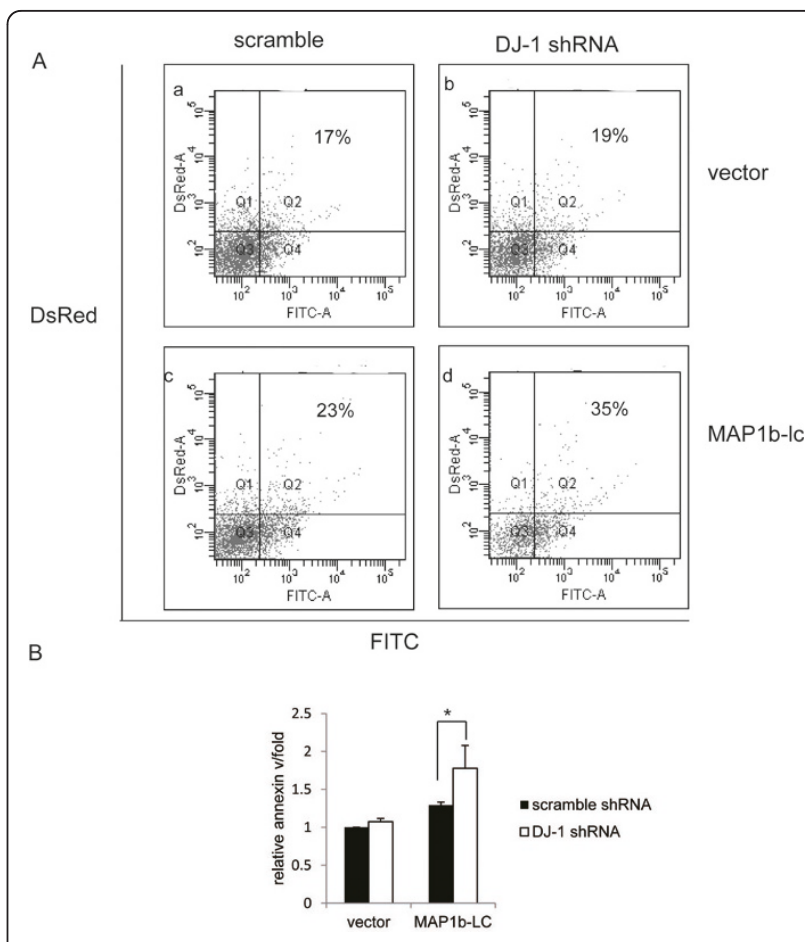

Figure 6 overburdened aggregation of MAP1b-LC in the DJ-1 KD SH-SY5Y cells leads to apoptosis. A, pCNDA3-MAP1b-LC or blank pcDNA3 were transfected into DJ-1 KD SH-SY5Y cells or scrambled controls. pDsRed2 was co-transfected to mark the transfected cells. Sixty hours later, cells were stained by Annexin-v and analyzed by flow cytometer. The data showed that MAP1b-LC can induce apoptosis (23\% Annexin- $v$ positive of the total transfected cells); the apoptosis cells increased to $35 \%$ in the DJ-1 KD cells compared to the scramble. B, The statistical analysis result showed that the apoptosis was increased when MAP1b-LC was introduced into DJ-1 KD SH-SY5Y cells (*, $p=0.04)$

SH-SY5Y cells could be inhibited by the overexpression of DJ-1.

Protein aggregation and insoluble inclusion bodies are one of the main causes for the pathogenesis of many neurodegenerative diseases [2]. MAP1b is essential for normal development of the murine nervous system [13] and its abnormal accumulation has been linked to neurodegenerative disease. Moreover, MAP1b-LC has been observed in the insoluble Lewy bodies in the brain of PD patients [14]. Allen et al. reported that MAP1b-LC accumulation could lead to the neuron death in GAN KO mouse [11]. Since DJ-1 has been shown to interact with MAP1b-LC and inhibit its aggregation, we hypothesized that the formation of MAP1b-LC aggregation may be suppressed by the molecular chaperone DJ-1.

Mutations of DJ-1 are thought to be loss function mutations that can lead to autosomal recessive familial PD. The most frequently DJ-1 mutant L166P fails to form dimers and monomeric mutant DJ-1 is unstable and degraded rapidly [23]. Therefore DJ-1 KD SH-SY5Y 


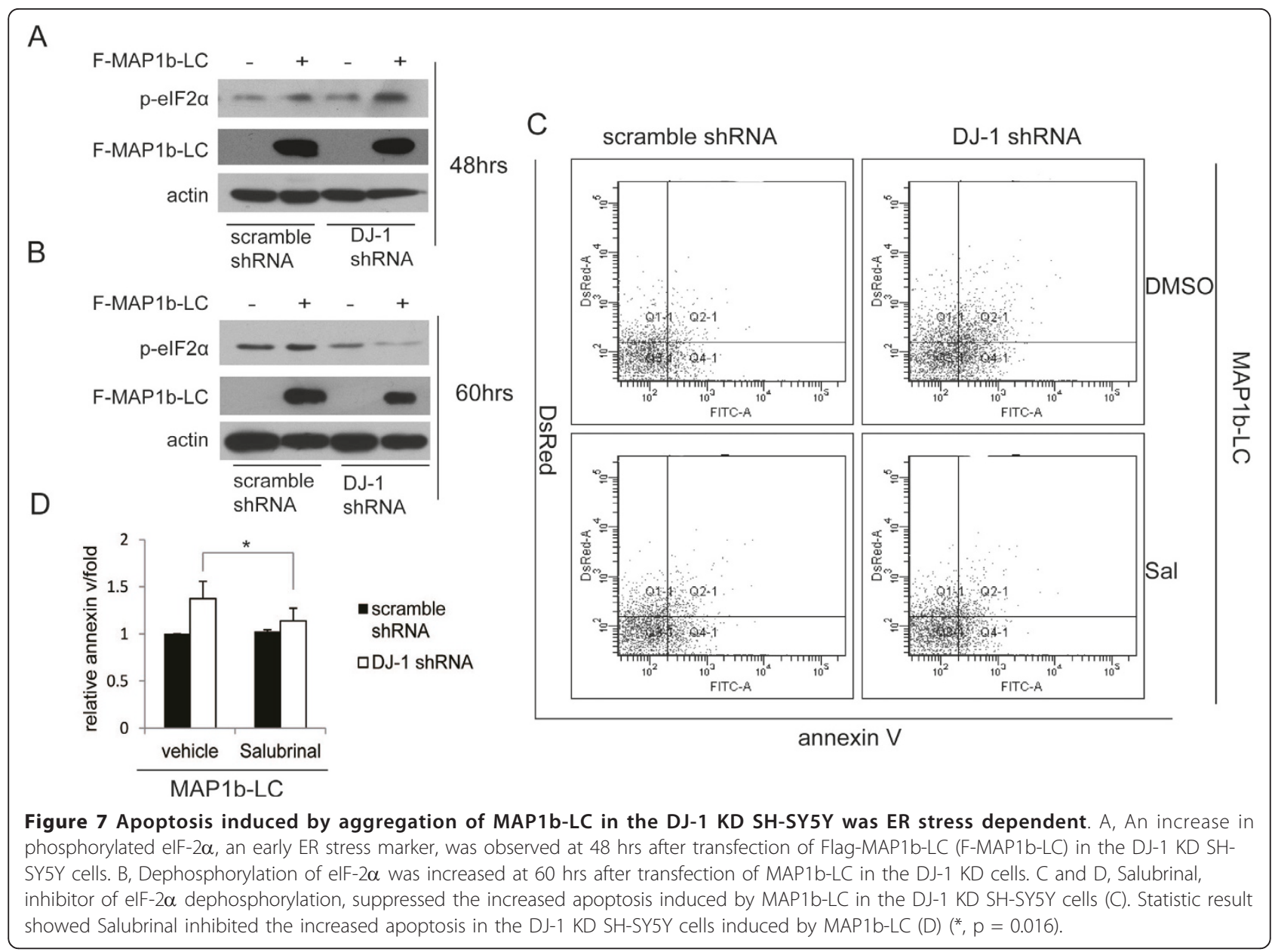

stable cell lines and DJ-1 KO mice were used to study the effect of DJ-1 on the aggregation of MAP1b-LC. In the DJ-1 KD cells, MAP1b-LC aggregation was increased compared with that of the scramble control cells. Moreover, the level of insoluble MAP1b-LC was also increased in the DJ-1 KO mice. So it implicated that DJ-1 may work as a molecular chaperone to control the normal state of MAP1b-LC and loss function of DJ1 may lead to increased aggregation of MAP1b-LC.

It has been proposed that the impairments of ubiquitin proteasome system (UPS) play an important role in the pathogenesis of PD. DJ-1 has also been reported to participate in regulating the activity of UPS [24,25]. It has been found that inhibition of MAP1b-LC ubiquitination leads to neuronal death in the GAN-null mice [11]. Therefore, we examined whether increased aggregation of MAP1b-LC results from the failure of its UPS dependent degradation. However, our results did not show any change of the ubiquitination of MAP1b-LC (Figure 5A). Neither was there any change of the total protein (soluble plus insoluble) level of MAP1b-LC (Figure $4 \mathrm{G}$ ). Moreover, there was no impairment of proteasome activity in DJ-1 KD cells and DJ-1 KO mice. All of these observations have indicated that the loss-offunction of DJ-1 does not affect the UPS dependent degradation of MAP1b-LC. The increase in insoluble MAP1b-LC in the DJ-1 KD cells and DJ-1 KO mice suggests that DJ-1 may work as a molecular chaperone to promote correct folding of MAP1b-LC or maintain the normal state of MAP1b-LC.

Protein aggregation has been implicated to play an important role in the pathogenesis of neurodegenerative diseases. Therefore, we analyzed whether excessive MAP1b-LC aggregation can cause cell apoptosis. The data suggested that overexpression of MAP1b-LC in the DJ-1 KD SH-SY5Y cells, which produced overburdened MAP1b-LC aggregates, increased cell apoptosis. It has been reported that abnormal protein aggregation can induce apoptosis mainly through the ER stress pathway [36]. Previous studies have implicated that phosphorylation of eIF2 $\alpha$ was a protective cell response to counteract the ER stress and the failure of the phosphorylation of elf2 $\alpha$ will lead to apoptosis $[28,29]$. Our results revealed that the enhancement of MAP1b-LC 
aggregation can induced more phosphorylated eIF2 $\alpha$ in DJ-1 KD cells than that in scramble controls. The persistent existence of MAP1b-LC aggregation increased dephosphorylated eIF $2 \alpha$ and led the cells to ER stress dependent apoptosis at 60 hrs. The fact that the eIF $2 \alpha$ dephosphorylation inhibitor Salubrinal can partially inhibit the apoptosis supported our hypothesis.

DJ-1 has been shown to be a molecular chaperone that can inhibit $\alpha$-synuclein aggregation $[20,21]$. However, Ramsey et. al showed that DJ-1-deficient mice had similar vulnerability to pathogenic Ala53Thr human $\alpha$ synuclein toxicity [37]. Based on our experimental results, we cannot make the conclusion that chaperone activity of DJ-1 is unrelated to alpha-synuclein aggregation. It is possible that compensatory mechanisms exist in DJ-1 null mice which act to mimic the function of DJ-1 protein just as the author of the paper claimed [37].

We observed increased aggregation of MAP1b-LC in DJ-1 KD SH-SY5Y cells, as well as an increased level of insoluble MAP1b-LC in DJ-1 KO mice. In contrast, two previous studies did not observe the formation of inclusion bodies in either adult or aged DJ-1 null mice $[38,39]$. It suggested that DJ-1 abolishment produced more MAP1b-LC aggregation in vitro and more insoluble MAP1b-LC in vivo. There are two potential explanations for the difference between our in vitro and in vivo experiments: 1) In vitro experiment showed the acute responses of the cells to MAP1b overexpression or DJ-1 $K D$, in contrast, the in vivo study showed the chronic responses of animals to DJ-1 KO; and 2) both environmental and genetic factors are responsible for the pathogenesis of PD. The absence of MAP1b aggregation in DJ-1 KO mice may be due to the absence of certain non-genetic factors such as aging or neurotoxins in our study.

\section{Conclusions}

In summary, we report that DJ-1 is a molecular chaperone that can inhibit the aggregation of MAP1b-LC in vitro as well as the formation of insoluble MAP1b-LC in vivo. Our findings have provided the first evidence that links DJ-1 deficiency to MAPs aggregation, which may improve our understanding regarding the role of DJ-1 in the pathogenesis of PD.

\section{Materials and methods Antibodies and Reagents}

The following antibodies were used: DJ-1 Monoclonal Antibody (3E8) (Assay Designs, ADI-KAM-SA100-E), mono- and polyubiquitinylated conjugates, monoclonal Antibody (FK2) (Biomol, BML-PW8810R), DJ-1 polyclonal antibody (Abcam, ab18257), Rabbit polyclonal antiFlag (F7425), mouse monoclonal anti-Flag (F1804), mouse monoclonal anti-beta-actin (A5441) (SigmaAldrich), mouse anti-HA (clone 12C5) (Covance, MMS101R), mouse anti-EGFP (Roche, 11814460001), Phospho-elF2alpha (Ser51) antibody (Cell Signaling, 9721), Goat polyclonal anti-MAP1b (c-20) (Santa Cruz Biotechnology, sc-8971). EIF-2 $\alpha$ inhibitor Salubrinal was purchased from Calbiochem. All the Chemicals were purchased from Sigma-Aldrich except noted elsewhere.

\section{Plasmid construction}

Human DJ-1 and MAP1B-LC cDNA were amplified from the human fetal brain cDNA library (Invitrogen) and ligated to the pcDNA3 vector with an $\mathrm{N}$ terminal HA tag and pCMV-3xflag (sigma), respectively. To knock down DJ-1, a DNA fragment and a scramble fragment were synthesized and ligated to the pSilencer-3.1Hygro (Ambion). The sequence of the inserted DJ-1 DNA fragments is GATCCGCTAAAGGAGCAGAGGAAATTTCAAGAGAATTTCCTCTGCTCCTTTA GTTTTTTGGAAA, and the scramble sequence is GATCCGATCTCTTCTGGTATTAGACTCAAGAG ATCTAATACCAGAAGAGATCTTTTTTGGAAA. All the constructions were confirmed by sequencing. PCRbased site directed mutagenesis was used to construct the L166P mutation of DJ-1, which was cloned to the pEGFP-C2 (Clontech).

\section{Cell culture and transfection}

HEK293T and SH-SY5Y cells were purchased from American Type Culture Collection and maintained in DMEM with $10 \%$ Fetal Bovine Serum and $100 \mathrm{U} / \mathrm{ml}$ penicillin/streptomycin. All the culture materials were purchased from Invitrogen. HEK293t cells were transfected by calcium phosphate precipitation and SH-SY5Y cells were transfected with lipofectamine 2000 (Invitrogen). Transfection efficiency was neutralized by cotransfected with a pRL-tk plasmid. Because DJ-1 L166P is unstable, the amount of the plasmid used for its transfection was 3 times more than that used for the control vector. pSilencer-Hygro-DJ-1 used for DJ-1 KD was transfected into SH-SY5Y and the stable clones were selected with 300ug/ml Hygromycin.

\section{Co-immunoprecipitation and Western blotting}

For co-immunoprecipitation, HEK293T cells or the mice brain were lysed in the buffer A containing $50 \mathrm{mM}$ Tris. $\mathrm{HCl} \mathrm{pH}$ 7.6, $150 \mathrm{mM} \mathrm{NaCl}, 0.5 \% \mathrm{NP}-40,1 \%$ sodium deoxycholate and protease inhibitor cocktail (Roche). To detect the ubiquitination of MAP1b-LC, the cells were harvested and boiled in the lysis buffer B (50 $\mathrm{mM}$ Tris. $\mathrm{HCl} \mathrm{pH} 7.6,150 \mathrm{mM} \mathrm{NaCl}, 1 \% \mathrm{NP}-40,1 \%$ sodium deoxycholate, $1 \% \mathrm{SDS}$ ) for $10 \mathrm{~min}$. Then the concentration of SDS in Buffer B was diluted to $0.1 \%$ by RIPA buffer. The lysates were pre-cleared with protein 
A sepharose (GE bioscience) for $30 \mathrm{~min}$. The supernatants were incubated with the primary antibody for 4 hours at $4^{\circ} \mathrm{C}$. Then protein A sepharose (GE Bioscience) was added and the mixture was further incubated for 2 $\mathrm{h}$ at $4^{\circ} \mathrm{C}$. For the Flag fusion protein IP, Flag M2 beads (Sigma-Aldrich) were used. The beads with bound proteins were washed for 6 times with lysis buffer and were boiled in 2X SDS sample buffer, then the samples were detected by immunoblotting.

For Western blotting the cells were lysed in RIPA buffer $(50 \mathrm{mM}$ Tris. $\mathrm{HCl} \mathrm{pH} 7.6,150 \mathrm{mM} \mathrm{NaCl}, 1 \% \mathrm{NP}-40$, $1 \%$ sodium deoxycholate, $0.1 \%$ SDS) with protease inhibitor cocktail (Roche). To separate the detergents soluble and insoluble proteins, the homogenized mice brain or the cells were lysed in $0.2 \%$ Triton X-100 lysis buffer (25 mM Tris. $\mathrm{HCl} \mathrm{pH} 7.6,150 \mathrm{mM} \mathrm{NaCl}, 0.2 \%$ Triton $\mathrm{X}-100,1 \%$ sodium deoxycholate) on ice for $20 \mathrm{~min}$. Triton X-100-soluble and -insoluble fractions were separated via centrifugation at 13,000 rpm for $15 \mathrm{~min}$ [20]. The samples were boiled in 2XSDS sample buffer and detected by immunoblotting. For all the western blot results, at least 3 independent experiments were done and the most representative result was shown.

\section{Immunofluorescence microscopy}

HEK293T or SH-SY5Y cells were grown on glass coverslips, fixed with $4 \%$ PFA, permeabilized with $0.2 \%$ Triton $\mathrm{X}-100$, and blocked with $20 \%$ goat serum or $5 \% \mathrm{BSA}$ in $0.2 \%$ PBST and then incubated with primary antibody. Cells were washed and Alexa 594 or Alexa 488 goat anti-mouse or rabbit IgG antibody (Invitrogen) was added. After washed 3 times using PBS, anti-fade mounting medium with DAPI (Vector Laboratory) was added and the stained cells were analyzed with a confocal microscopy (Leica SP5).

\section{Apoptosis detection by Annexin V assay}

Cells were seeded in 6-well plates and transfected with the indicated plasmids and pDsRed 2 for 48 hours. Then cells were stained with Annexin V using the Annexin VFITC apoptosis detection kit (BD bioscience) as the instructions of the manufacturer. Cells $(30,000 /$ treatment) were analyzed using a flow cytometer (Becton Dickinson LSR II).

\section{Animal studies}

The DJ-1 knock-out mice were kindly provided from Dr. Jie Shen (Harvard Medical School) [40] and crossed with C57bl/6 mice at least 6 generations after arriving in our lab. Throughout the experiments, the animals were kept in stainless-steel cages in a controlled environment (22$25^{\circ} \mathrm{C}, 40-60 \%$ relative humidity, 12 -h light-dark cycle), with food and water available freely. All animal experiments were performed in accordance with guidelines of the laboratory animal ethical standards of Shanghai Jiao Tong University School of medicine. For animal studies, in each group 3 littermate mice brains were used, which was defined as one independent experiment. Statistical analyses were conducted on the results from three independent experiments.

\section{Statistical analysis}

Paired or unpaired Student's t-test was used for statistical analyses. Statistical significance was set at a $P$ value of less than 0.05 and there was no statistical correction was used for all the values.

\section{List of Abbreviations}

PD: Parkinson's disease; MAP1b LC: microtubule associated protein 1b; ER endoplasmic reticulum; KD: Knocking down; KO: Knock out; SDS-PAGE: sodium dodecyl sulfate polyacrylamide gel electrophoresis; GST: GlutathioneS-transferase; UPS: ubiquitin proteasome system.

\section{Acknowledgements}

We thank Dr. Jie Shen of Harvard medical school for her kindly providing of the DJ-1 Knock-out Mice. This work was supported by the National Program of Basic Research (2007CB947900, 2010CB945200, 2011CB504104) of China, the Natural Science Fund (30700888, 30770732, 30872729, 30971031), Key Discipline Program of Shanghai Municipality (S30202), Shanghai Key Project of Basic Science Research (10411954500), Shanghai Pujiang Program (08PJ1407900) and Program for Outstanding Medical Academic Leader of Shanghai $(\sqcup$ 06003).

\section{Author details}

'Laboratory of Neurodegenerative Diseases \& key Laboratory of Stem Cell Biology, Institute of Health Science, Shanghai Institutes for Biological Sciences, Chinese Academy of Science \& Shanghai Jiao Tong University School of Medicine. Shanghai 200025, China. ${ }^{2}$ Department of Neurology \& Institute of Neurology, Ruijin Hospital Affiliated to Shanghai Jiao Tong University School of Medicine. Shanghai 200025, China. ${ }^{3}$ Institut Pasteur of Shanghai, Shanghai Institutes for Biological Sciences, Chinese Academy of Science, Shanghai 200025, China.

\section{Authors' contributions}

ZQW designed and performed the experiments. YZ contributed to the protein expression and purification. SZ, XR and XYW helped to perform the animal studies. QQG helped to perform the flow cytometry analysis and YYT provided essential advice to the project. SDC and JQD supervised the project and edited the manuscript. All authors read and approved the final manuscript.

\section{Competing interests}

The authors declare that they have no competing interests.

Received: 9 September 2010 Accepted: 6 June 2011

Published: 6 June 2011

\section{References}

1. Tanner CM, Goldman SM: Epidemiology of Parkinson's disease. Neurol Clin 1996, 14:317-335.

2. Ross CA, Poirier MA: Protein aggregation and neurodegenerative disease. Nat Med 2004, 10(Suppl):S10-17.

3. Kopito RR: Aggresomes, inclusion bodies and protein aggregation. Trends Cell Biol 2000, 10:524-530

4. Ross CA, Poirier MA: Opinion: What is the role of protein aggregation in neurodegeneration? Nat Rev Mol Cell Biol 2005, 6:891-898.

5. Taylor JP, Hardy J, Fischbeck KH: Toxic proteins in neurodegenerative disease. Science 2002, 296:1991-1995.

6. Goldman JE, Yen SH: Cytoskeletal protein abnormalities in neurodegenerative diseases. Ann Neurol 1986, 19:209-223. 
7. Cairns NJ, Lee VM, Trojanowski JQ: The cytoskeleton in neurodegenerative diseases. J Pathol 2004, 204:438-449.

8. Goedert M: Tau protein and the neurofibrillary pathology of Alzheimer's disease. Ann N Y Acad Sci 1996, 777:121-131.

9. Dawson TM, Dawson VL: Molecular pathways of neurodegeneration in Parkinson's disease. Science 2003, 302:819-822.

10. Lu R, Wang H, Liang Z, Ku L, O'Donnell WT, Li W, Warren ST, Feng Y: The fragile $\mathrm{X}$ protein controls microtubule-associated protein $1 \mathrm{~B}$ translation and microtubule stability in brain neuron development. Proc Natl Acad Sci USA 2004, 101:15201-15206.

11. Allen E, Ding J, Wang W, Pramanik S, Chou J, Yau V, Yang Y: Gigaxonincontrolled degradation of MAP1B light chain is critical to neuronal survival. Nature 2005, 438:224-228.

12. Gonzalez-Billault C, Jimenez-Mateos EM, Caceres A, Diaz-Nido J, Wandosell F, Avila J: Microtubule-associated protein 1B function during normal development, regeneration, and pathological conditions in the nervous system. J Neurobiol 2004, 58:48-59.

13. Takei Y, Kondo S, Harada A, Inomata S, Noda T, Hirokawa N: Delayed development of nervous system in mice homozygous for disrupted microtubule-associated protein 1B (MAP1B) gene. J Cell Biol 1997. 137:1615-1626.

14. Jensen PH, Islam K, Kenney J, Nielsen MS, Power J, Gai WP: Microtubuleassociated protein $1 \mathrm{~B}$ is a component of cortical Lewy bodies and binds alpha-synuclein filaments. J Biol Chem 2000, 275:21500-21507.

15. Bonifati V, Rizzu P, van Baren MJ, Schaap O, Breedveld GJ, Krieger E, Dekker MC, Squitieri F, Ibanez P, Joosse M, et al: Mutations in the DJ-1 gene associated with autosomal recessive early-onset parkinsonism. Science 2003, 299:256-259.

16. Bonifati V, Oostra BA, Heutink P: Linking DJ-1 to neurodegeneration offers novel insights for understanding the pathogenesis of Parkinson's disease. J Mol Med 2004, 82:163-174.

17. Bandopadhyay R, Kingsbury AE, Cookson MR, Reid AR, Evans IM, Hope AD, Pittman AM, Lashley T, Canet-Aviles R, Miller DW, et al: The expression of DJ-1 (PARK7) in normal human CNS and idiopathic Parkinson's disease. Brain 2004, 127:420-430.

18. Lee SJ, Kim SJ, Kim IK, Ko J, Jeong CS, Kim GH, Park C, Kang SO, Suh PG, Lee HS, Cha SS: Crystal structures of human DJ-1 and Escherichia coli Hsp31, which share an evolutionarily conserved domain. J Biol Chem 2003, 278:44552-44559.

19. Tao $X$, Tong L: Crystal structure of human DJ-1, a protein associated with early onset Parkinson's disease. J Biol Chem 2003, 278:31372-31379.

20. Shendelman S, Jonason A, Martinat C, Leete T, Abeliovich A: DJ-1 is a redox-dependent molecular chaperone that inhibits alpha-synuclein aggregate formation. PLOS Biol 2004, 2:362.

21. Batelli S, Albani D, Rametta R, Polito L, Prato F, Pesaresi M, Negro A, Forloni G: DJ-1 modulates alpha-synuclein aggregation state in a cellular model of oxidative stress: relevance for Parkinson's disease and involvement of HSP70. PLOS One 2008, 3:1884.

22. Jin J, Li GJ, Davis J, Zhu D, Wang Y, Pan C, Zhang J: Identification of novel proteins associated with both alpha-synuclein and DJ-1. Mol Cell Proteomics 2007, 6:845-859

23. Moore DJ, Zhang L, Dawson TM, Dawson VL: A missense mutation (L166P) in DJ-1, linked to familial Parkinson's disease, confers reduced protein stability and impairs homo-oligomerization. J Neurochem 2003, 87:1558-1567.

24. Xiong H, Wang D, Chen L, Choo YS, Ma H, Tang C, Xia K, Jiang W, Ronai Z, Zhuang X, Zhang Z: Parkin, PINK1, and DJ-1 form a ubiquitin E3 ligase complex promoting unfolded protein degradation. J Clin Invest 2009, 119:650-660.

25. Yokota T, Sugawara K, Ito K, Takahashi R, Ariga H, Mizusawa H: Down regulation of DJ-1 enhances cell death by oxidative stress, ER stress, and proteasome inhibition. Biochem Biophys Res Commun 2003, 312:1342-1348.

26. Demo SD, Kirk CJ, Aujay MA, Buchholz TJ, Dajee M, Ho MN, Jiang J, Laidig GJ, Lewis ER, Parlati F, et al: Antitumor activity of PR-171, a novel irreversible inhibitor of the proteasome. Cancer Res 2007, 67:6383-6391.

27. Xu C, Bailly-Maitre B, Reed JC: Endoplasmic reticulum stress: cell life and death decisions. J Clin Invest 2005, 115:2656-2664.

28. Boyce M, Bryant KF, Jousse C, Long K, Harding HP, Scheuner D, Kaufman RJ, Ma D, Coen DM, Ron D, Yuan J: A selective inhibitor of elF2alpha dephosphorylation protects cells from ER stress. Science 2005, 307:935-939
29. Harding HP, Zhang Y, Bertolotti A, Zeng H, Ron D: Perk is essential for translational regulation and cell survival during the unfolded protein response. Mol Cell 2000, 5:897-904.

30. Mitsumoto A, Nakagawa Y: DJ-1 is an indicator for endogenous reactive oxygen species elicited by endotoxin. Free Radic Res 2001, 35:885-893.

31. Menzies FM, Yenisetti SC, Min KT: Roles of Drosophila DJ-1 in survival of dopaminergic neurons and oxidative stress. Curr Biol 2005, 15:1578-1582.

32. Meulener M, Whitworth AJ, Armstrong-Gold CE, Rizzu P, Heutink P, Wes PD, Pallanck LJ, Bonini NM: Drosophila DJ-1 mutants are selectively sensitive to environmental toxins associated with Parkinson's disease. Curr Biol 2005, 15:1572-1577.

33. Taira T, Saito Y, Niki T, Iguchi-Ariga SM, Takahashi K, Ariga H: DJ-1 has a role in antioxidative stress to prevent cell death. EMBO Rep 2004, 5:213-218

34. Martinat C, Shendelman S, Jonason A, Leete T, Beal MF, Yang L, Floss T, Abeliovich A: Sensitivity to oxidative stress in DJ-1-deficient dopamine neurons: an ES- derived cell model of primary Parkinsonism. PLoS Biol 2004, 2:327.

35. Andres-Mateos E, Perier C, Zhang L, Blanchard-Fillion B, Greco TM, Thomas B, Ko HS, Sasaki M, Ischiropoulos H, Przedborski S, et al: DJ-1 gene deletion reveals that $D J-1$ is an atypical peroxiredoxin-like peroxidase. Proc Natl Acad Sci USA 2007, 104:14807-14812.

36. Scheper W, Hoozemans JJ: Endoplasmic reticulum protein quality control in neurodegenerative disease: the good, the bad and the therapy. Curr Med Chem 2009, 16:615-626.

37. Ramsey CP, Tsika E, Ischiropoulos H, Giasson BI: DJ-1 deficient mice demonstrate similar vulnerability to pathogenic Ala53Thr human alphasyn toxicity. Hum Mol Genet 19:1425-1437.

38. Chen L, Cagniard B, Mathews T, Jones S, Koh HC, Ding Y, Carvey PM, Ling Z, Kang UJ, Zhuang X: Age-dependent motor deficits and dopaminergic dysfunction in DJ-1 null mice. J Biol Chem 2005, 280:21418-21426.

39. Yamaguchi $\mathrm{H}$, Shen J: Absence of dopaminergic neuronal degeneration and oxidative damage in aged DJ-1-deficient mice. Mol Neurodegener 2007, 2:10

40. Goldberg MS, Pisani A, Haburcak M, Vortherms TA, Kitada T, Costa C, Tong Y, Martella G, Tscherter A, Martins A, et al: Nigrostriatal dopaminergic deficits and hypokinesia caused by inactivation of the familial Parkinsonism-linked gene DJ-1. Neuron 2005, 45:489-496.

doi:10.1186/1750-1326-6-38

Cite this article as: Wang et al.: DJ-1 can inhibit microtubule associated protein 1 B formed aggregates. Molecular Neurodegeneration 2011 6:38.

\section{Submit your next manuscript to BioMed Central and take full advantage of:}

- Convenient online submission

- Thorough peer review

- No space constraints or color figure charges

- Immediate publication on acceptance

- Inclusion in PubMed, CAS, Scopus and Google Scholar

- Research which is freely available for redistribution 